\title{
Epigallocatechin-3-gallate hinders metabolic coupling to suppress colorectal cancer malignancy through targeting aerobic glycolysis in cancer-associated fibroblasts
}

\author{
SHUHAI CHEN, MASAAKI NISHI, YUJI MORINE, MITSUO SHIMADA, TAKUYA TOKUNAGA, \\ HIDEYA KASHIHARA, CHIE TAKASU, SHINICHIRO YAMADA and YUMA WADA
}

Department of Surgery, Institute of Biomedical Sciences, Tokushima University, Tokushima 770-8503, Japan

Received September 3, 2021; Accepted November 29, 2021

DOI: 10.3892/ijo.2022.5309

\begin{abstract}
In recent times, researchers working on tumor metabolism have paid increasing attention to the tumor microenvironment. Emerging evidence has confirmed that epigenetic modifications of cancer-associated fibroblasts (CAFs) alters the characteristics of glucose metabolism to achieve a symbiotic relationship with the cancer cells. Epigallocatechin-3-gallate (EGCG) exerts anti-tumor effects via a variety of mechanisms, although the underlying mechanism that accounts for the effects of EGCG on glucose metabolic alterations of CAFs have yet to be elucidated. In the present study, through co-culture with colorectal cancer (CRC) cells, human intestinal fibroblasts were transformed into CAFs, and exhibited enhanced aerobic glycolysis. Induced CAFs were able to enhance the proliferation, migration and invasion of CRC cells in vitro. EGCG treatment led to direct inhibition of the proliferation and migration of CRC cells; furthermore, EGCG treatment of CAFs suppressed their tumor-promoting capabilities by inhibiting their glycolytic activity. Blocking the lactic acid efflux of CAFs with a monocarboxylate transporter 4 (MCT4) inhibitor or through silencing MCT4 could also suppress their tumor-promoting capabilities, indicating that lactate fulfills an important role in the metabolic coupling that occurs between CAFs and cancer cells. Taken together, the results of the
\end{abstract}

Correspondence to: Dr Masaaki Nishi, Department of Surgery, Institute of Biomedical Sciences, Tokushima University, 3-18-15 Kuramoto-cho, Tokushima 770-8503, Japan

E-mail: nishi.masaaki@tokushima-u.ac.jp

Abbreviations: $\alpha$-SMA, $\alpha$-smooth muscle actin; ACTA2, actin $\alpha-2$; CAF, cancer-associated fibroblast; CM, conditioned medium; CRC, colorectal cancer; EGCG, epigallocatechin-3-gallate; FAP, fibroblast activation protein; HIFs, human intestinal fibroblasts; MCT, monocarboxylate transporter; TBST, Tris Buffered Saline with Tween-20; TME, tumor microenvironment; PFK, phosphofructokinase

Keywords: cancer-associated fibroblasts, epigallocatechin-3-gallate, aerobic glycolysis, reverse Warburg effect, colorectal cancer present study showed that EGCG targeting of the metabolism of tumor stromal cells provided a safe and effective strategy of anti-cancer therapy.

\section{Introduction}

Colorectal cancer (CRC), one of the most common and aggressive human malignancies, causes numerous cancer-associated deaths worldwide annually $(1,2)$. The shift to aerobic glycolytic metabolism in cancer cells has been widely studied and updated in the well-known 'hallmarks of cancer', as described by Hanahan and Weinberg (3). However, the current view is that research on cancer metabolism should not be restricted to cancer cells (4). The tumor microenvironment (TME) is composed of several cell types that are allocated the constant supplies of nutrients required for tumor survival and progression (5). Emerging evidence has confirmed that cancer-associated fibroblasts (CAFs) undergo epigenetic modifications in molecules associated with glucose metabolism to achieve a symbiotic relationship with cancer cells (6-8). CAFs with enhanced glycolytic features subsequently secrete high amounts of pyruvate and lactate, which are in turn used by cancer cells as new sources of energy (9). This new concept is referred to as the 'reverse Warburg effect', and this indicates a potential therapeutic strategy for cancer $(6,10-12)$. Recently, chip-based 3D co-culture technology has been applied to simulate the characteristics of the metabolic coupling and 'reverse Warburg effect' between CRC cells and fibroblasts in vitro (13).

Green tea is a popular beverage worldwide, and it has long been described to possess several health benefits (14). As a safe and the most powerful ingredient in green tea (15), epigallocatechin-3-gallate (EGCG) possesses high chemopreventative properties, and has been proposed as a possible chemotherapeutic treatment against numerous types of cancer, including hepatocellular carcinoma (16), CRC (17), breast cancer (18) and B lymphoma (19). It has been concluded that it is safe for humans to take the amounts equivalent to the EGCG content in 8-16 cups of green tea once a day for 4 weeks (20). To date, various mechanisms have been proposed to explain the cancer-preventative effects of EGCG, including the upregulation of tumor suppressor genes such as p53 (21), and 
the modulation of cell signaling pathways, such as nuclear factor- $\kappa \mathrm{B}$ (22) and JAK/STAT3 (23). More recently, EGCG has been shown to inhibit cell growth and induce apoptosis through targeting the enhanced aerobic glycolytic pathway via directly inhibiting phosphofructokinase (PFK) activity in cancer cells $(18,24)$.

However, to the best of our knowledge, there are no previous studies assessing the effects of EGCG on glucose metabolic alterations involving CAFs in the TME. The aim of the present study was to investigate the hypothesis that EGCG may intervene in the metabolic coupling between CAFs and CRC cells through inhibiting aerobic glycolytic activity in CAFs, thereby suppressing the malignancy of CRC cells in vitro. The findings obtained highlight a novel anti-tumor strategy of EGCG, in which it targets the glucose metabolism of stromal cells in the TME, providing further insights into elucidating the underlying mechanism of metabolic coupling between CRC cells and stromal cells.

\section{Materials and methods}

Cell culture and reagents. Human colorectal carcinoma cell lines HCT-116 (CCL-247) and HT-29 (HTB-38) were purchased from ATCC and cultured in McCoy's 5A (Thermo Fisher Scientific, Inc.) supplemented with 10\% FBS (Thermo Fisher Scientific, Inc.) and a $1 \%$ penicillin-streptomycin solution (Thermo Fisher Scientific, Inc.). Human intestinal fibroblasts (HIFs) were obtained from ScienCell Research Laboratories, Inc. (cat. no. 2920) and cultured in complete fibroblast medium (cat. no. 2301; ScienCell Research Laboratories). Cells were cultured at $37^{\circ} \mathrm{C}$ in a humidified incubator with $5 \% \mathrm{CO}_{2}$ and normal oxygen saturation, and were used for experiments during the exponential growth phase under mycoplasma-free conditions. All cell lines used in the present study were authenticated by short tandem repeat profiling by the companies they were purchased from, were aliquoted and frozen in liquid nitrogen immediately upon receipt, and used for the present experiments within 6 months of thawing. EGCG was purchased from Funakoshi Co., Ltd. (cat. no. E-5737) and bindarit (2-methyl-2-[(1-[phenylmethyl]-1H-indazol-3yl)methoxy] propanoic acid) was supplied by MedChemExpress.

Cell co-culture and conditioned medium (CM) collection. To induce CAFs, HIFs were co-cultured with HCT-116 or HT-29 cells at a ratio of 1:3 using Falcon ${ }^{\circledR}$ Permeable supports for six-well plates with $0.4-\mu \mathrm{m}$ pores (cat. no. 353090; Corning, Inc.) in an indirect contact manner for 3 days, sharing the same media; DMEM high glucose $(4.5 \mathrm{~g} / \mathrm{l})$, pyruvate $(110 \mathrm{mg} / \mathrm{l})$ (cat. no. 11995065; Thermo Fisher Scientific, Inc.) supplemented with $10 \%$ FBS. Subsequently, the cancer cells were discarded from the co-culture system, and the medium was replaced with fresh serum-free DMEM for the monoculture of induced CAFs. After a further $48 \mathrm{~h}$ of culture, the supernatant was collected, centrifuged $(500 \mathrm{x} \mathrm{g}$ at room temperature for $20 \mathrm{~min}$ ), and filtered using a $0.2-\mu \mathrm{m}$ filter to remove the cell debris. For EGCG or bindarit treatment, different concentrations (as described in results section) of reagents were added to fresh DMEM supplemented with $10 \%$ FBS to induce CAFs for a further $24 \mathrm{~h}$ after discarding the cancer cells, and before further replacement of new medium to generate CM. To generate HIF-CM, HIFs were cultured without cancer cells, and the same procedure as that described above was employed. For CAF-siRNA-MCT4-CM and CAF-siRNA-NC-CM, induced CAFs were first transfected with monocarboxylate transporter (MCT) 4 siRNA or Select Negative Control \#1 siRNA respectively, and the remaining steps in the procedure were the same as those described above. All CM obtained from the above groups was stored at $-80^{\circ} \mathrm{C}$. For the further treatments of cancer cells in the subsequent experiments, $\mathrm{CM}$ was warmed to room temperature and added to fresh medium at a ratio of 1:1.

Immunofluorescence staining. Cells were seeded in the Nunc Lab-Tek II Chamber Slide system (cat. no. 154526PK; Thermo Fisher Scientific, Inc.) until they had adhered, washed twice with PBS, and fixed with $4 \%$ paraformaldehyde (cat. no. 163-20145; Fujifilm) at $4^{\circ} \mathrm{C}$ for $30 \mathrm{~min}$. After permeabilization with $0.1 \%$ Triton $\mathrm{X}-100$ (cat. no. HFH10; Thermo Fisher Scientific, Inc.) for $5 \mathrm{~min}$ at room temperature, all slides were incubated with $3 \%$ bovine serum albumin (BSA) diluted from a MACS BSA stock solution (cat. no. 130-091-376; Miltenyi Biotec) in PBS for $60 \mathrm{~min}$ at room temperature. Subsequently, the slides were incubated with an anti- $\alpha$ smooth muscle actin ( $\alpha$-SMA) antibody (dilution, 1:300; cat. no. no. ab7817; Abcam) or anti-fibroblast activation protein (anti-FAP) (1:100; cat. no. ab207178, Abcam) at $4^{\circ} \mathrm{C}$ overnight. The following day, the slides were washed three times with PBS and incubated with Alexa Fluor 488-conjugated goat anti-rabbit IgG $(\mathrm{H}+\mathrm{L})$ cross-adsorbed secondary antibody (1:500; cat. no. A-11008, Thermo Fisher Scientific, Inc.) for FAP or Alexa Fluor 594-conjugated goat anti-mouse $\operatorname{IgG}(\mathrm{H}+\mathrm{L})$ cross-adsorbed secondary antibody (1:500; cat. no. A-11005, Thermo Fisher Scientific, Inc.) for $\alpha$-SMA for $1 \mathrm{~h}$ at $37^{\circ} \mathrm{C}$ in the dark. After three washes with PBS protected from light, ProLong ${ }^{\mathrm{TM}}$ Gold Antifade Mount with DAPI (cat. no. P36931; Thermo Fisher Scientific, Inc.) was used to stain the nuclei for $10 \mathrm{~min}$ in the dark. Finally, the slides were observed and imaged under a fluorescence microscope (magnification, x100; BZ-X700; Keyence Corporation). For negative controls, the primary antibody was replaced with BSA.

Cell proliferation assay. HCT-116 or HT-29 cells were seeded at a density of $1 \times 10^{4}$ cells/well into 96-well plates until they had adhered to the plates. After washing with PBS, the indicated $\mathrm{CM}$ was added to each group for $48 \mathrm{~h}$ of culture. Subsequently, the medium for each group was replaced with fresh medium containing $10 \%$ (v/v) Cell Counting Kit-8 (CCK-8) solution (Dojindo Molecular Technologies, Inc.), the plates were cultured for a further $2 \mathrm{~h}$, and the extent of cell proliferation was analyzed by measuring the absorbance values at $450 \mathrm{~nm}$ with a microplate reader (SpectraMax i3; Molecular Devices, LLC), in accordance with the manufacturer's instructions. To examine the direct effects of EGCG on cancer cells and the cytotoxicity of EGCG on normal cells, HCT-116 and HT-29 cells and HIFs were seeded at a density of $5 \times 10^{3}$ cells/well into 96-well plates and treated with different concentrations of EGCG. The proliferative status of the cells was observed every $24 \mathrm{~h}$ between days 1 and 3 using CCK- 8 solution, as described above. 
Wound healing assay. For wound healing assays, HCT-116 or HT-29 cells were seeded at a density of $7 \times 10^{5}$ cells/well into six-well plates and incubated overnight to form a $90 \%$ confluent monolayer. Subsequently, a 200- $\mu 1$ pipette tip was used to scratch a wound through the entire center of each well. After washing with PBS, the cells in each group were cultured with EGCG, bindarit or the indicated CM in the absence of FBS for $36 \mathrm{~h}$. The areas of the wounds were observed, and images were captured using a light microscope (magnification, $\mathrm{x} 40$; DP22-CU; Olympus Corporation) at 0 and $36 \mathrm{~h}$ after scratching. The cell migration rates were calculated using ImageJ v1.46r software (National Institutes of Health) according to the following equation: Relative migration rate $=[$ width $(0 \mathrm{~h})$-width $(36 \mathrm{~h})] /$ width $(0 \mathrm{~h}) \times 100 \%$.

Migration and invasion assay. A 24-well Transwell system with $8.0-\mu \mathrm{m}$ pores (Corning, Inc.) was used for the migration and invasion assays. Serum-starved cancer cells at a density of $7 \times 10^{4} /$ well were seeded into the upper chamber in a $100 \mu \mathrm{l}$ volume suspension. After cell attachment, the culture medium was changed to the indicated $\mathrm{CM}$ for each group. The final FBS concentration was 5\% in the upper chambers and $10 \%$ in the lower chambers. After a subsequent period of incubation ( $36 \mathrm{~h}$ for migration assay; $60 \mathrm{~h}$ for invasion assay), the upper chambers were fixed with $100 \%$ methanol (cat. no. 131-01826; Fujifilm) for $20 \mathrm{~min}$ and then stained with $1 \%$ crystal violet (cat. no. 031-04852; Fujifilm) for a further $20 \mathrm{~min}$ at room temperature, and non-migrated cells were removed using cotton swabs. Subsequently, images were captured, and the number of remaining cells were calculated and quantified in 5 random fields of view under a light microscopic (magnification, x100) equipped with a digital camera (Olympus Corporation). For the invasion assays, the upper chambers of the Transwell system were coated with Matrigel $^{\mathrm{TM}}(0.5 \mathrm{mg} / \mathrm{ml}$ dilatated in DMEM; Corning, Inc.) overnight at $37^{\circ} \mathrm{C}$.

Reverse transcription-quantitative (RT-q)PCR. Total RNA was extracted using an RNeasy Mini kit (Qiagen $\mathrm{GmbH}$, Hilden, Germany), and its concentration was measured using a spectrophotometer (NanoDrop ${ }^{\mathrm{TM}}$ 2000; Thermo Fisher Scientific, Inc.) according to the manufacturer's instructions. Subsequently, $2.5 \mu \mathrm{g}$ RNA was reverse-transcribed into cDNA in a total reaction system of $50 \mu \mathrm{l}$ using an Applied Biosystems $^{\mathrm{TM}}$ High-Capacity cDNA Reverse Transcription kit (Thermo Fisher Scientific, Inc.) in accordance with the manufacturer's instructions. Subsequently, an Applied Biosystems StepOnePlus Real-Time PCR system (Thermo Fisher Scientific, Inc.) was used to perform TaqMan qPCR using the following thermocycling conditions: Initial denaturation at $95^{\circ} \mathrm{C}$ for $3 \mathrm{~min} ; 40$ cycles of denaturation $\left(95^{\circ} \mathrm{C}\right.$ for $30 \mathrm{sec})$, annealing $\left(58^{\circ} \mathrm{C}\right.$ for $\left.30 \mathrm{sec}\right)$ and extension $\left(72^{\circ} \mathrm{C}\right.$ for $45 \mathrm{sec})$; and final extension at $72^{\circ} \mathrm{C}$ for $10 \mathrm{~min}$. The following TaqMan gene expression assays were used: ACTA2 (assay ID, Hs00426835_g1) FAP (assay ID, Hs00990791_m1), interleukin-6 (IL-6) (assay ID, Hs00985639_m1), PFK (assay ID, Hs00737347_m1), and SLC16A3 (MCT4) (assay ID, Hs00358829_m1). A GAPDH assay (assay ID, 4326317E; Thermo Fisher Scientific, Inc.) was used as the internal control to normalize the raw data. The $2^{-\Delta \Delta C q}$ method (25) was used for data analysis, and the results are presented as the fold changes in the relative mRNA expression for each experimental group compared with that in the control group.

MCT4 silencing through siRNA transfection. Induced CAFs were transfected with $10 \mathrm{pmol} / \mathrm{ml}$ SLC16A3 (MCT4) siRNA (cat. no. 4390824; assay ID: s17417; Thermo Fisher Scientific, Inc.) or Select Negative Control \#1 siRNA (cat. no. 4390843; Thermo Fisher Scientific, Inc.) with $3 \mu 1 / \mathrm{ml}$ Invitrogen ${ }^{\mathrm{TM}}$ Lipofectamine RNAiMax Transfection Reagent (cat. no. 13778075; Thermo Fisher Scientific Inc.) for $12 \mathrm{~h}$ according to the manufacturer's instructions. The efficiency of gene silencing was verified through performing RT-qPCR and western blotting.

Lactate assay. The supernatant was collected using the same method as that used for $\mathrm{CM}$ after culturing the indicated cells for 6,12 or $24 \mathrm{~h}$. A lactate assay kit (cat. no. MAK064; Sigma-Aldrich) was used to detect the concentration of lactate in the supernatant. Briefly, supernatants and standard samples were diluted with lactate assay buffer according to the manufacturer's instructions and added to a 96-well plate. After a 30 min incubation with $50 \mu \mathrm{l}$ master reaction mix at room temperature, the absorbance values at $570 \mathrm{~nm}$ were measured with a microplate reader, and the lactate concentrations of samples were calculated according to a standard curve constructed using standard samples. The relative secretion of lactate was normalized to the cell number.

Western blot analysis. Freshly prepared RIPA lysis buffer (Thermo Fisher Scientific, Inc.) containing 1\% protease inhibitor cocktail (cat. no. P8849; Sigma-Aldrich) and $1 \%$ phosphatase inhibitor cocktail 3 (cat. no. P0044; Sigma-Aldrich) was used to extract the total protein, and the protein concentration was measured using a BCA kit (Thermo Fisher Scientific, Inc.). Equal amounts of protein $(10 \mu \mathrm{g})$ were separated by SDS-PAGE (10\% gels) and transferred to PVDF membranes with $0.45-\mu \mathrm{m}$ pores (Sigma-Aldrich; Merck KGaA). After washing with Tris Buffered Saline with Tween-20 (TBST) (cat. no. 9997; Cell Signaling Technology, Inc.) for $10 \mathrm{~min}$ at room temperature, $5 \%$ skimmed milk was used as the blocking solution to incubate the membranes under gentle shaking for $90 \mathrm{~min}$ at room temperature. The membranes were washed with TBST 4 times (10 min each) and then incubated with anti- $\alpha$-SMA antibody (dilution, 1:2,000; cat. no. ab7817, Abcam), anti-FAP (1:3,000; cat. no. ab28244; Abcam), anti-PFK (1:20,000; cat. no. ab204131; Abcam), anti-MCT4 (1:2,000; cat. no. 22787-1-AP; ProteinTech Group, Inc.) or anti- $\beta$-actin (1:2,000; cat. no. 4970; Cell Signaling Technology, Inc.) as the primary antibodies overnight at $4^{\circ} \mathrm{C}$. The following day, after washing with TBST 4 times (10 min each), anti-rabbit IgG, HRP-linked (1:3,000; cat. no. 7074; Cell Signaling Technology, Inc.) and anti-mouse IgG, HRP-linked (1:3,000; cat. no. 7076; Cell Signaling Technology, Inc.) were used as the secondary antibodies, according to the species of the primary antibodies, to incubate the membranes for $1 \mathrm{~h}$ at room temperature. Protein signals were visualized using ECL Prime Western Blotting Detection Reagents (cat. no. RPN2232; Cytiva). 
Statistical analysis. All data are presented as the mean \pm standard deviation of at least three biological repeats. GraphPad Prism v7.0 software (GraphPad Software, Inc.) and ImageJ v1.46r software were used for the statistical analysis and construction of the graphs. An unpaired Student's t-test or a Mann-Whitney U test were used for comparisons between two groups. Differences between multiple groups were analyzed using an ANOVA followed by a Tukey's post hoc test. $\mathrm{P}<0.05$ (two-sided) was considered to indicate a statistically significant difference.

\section{Results}

Fibroblasts co-cultured with tumor cells exhibit a CAF phenotype with enhanced features of aerobic glycolysis. After co-culture with the HCT-116 and HT-29 CRC cell lines, HIFs exhibited higher gene expression levels of the CAF markers ACTA2, FAP and IL- 6 (Fig. 1A). The western blotting results showed that the expression levels of $\alpha$-SMA and FAP, the two most widely used markers for the identification of CAFs, were upregulated in HIFs after co-culture (Fig. 1B). Moreover, the results from the immunofluorescence staining experiments confirmed the upregulation of $\alpha$-SMA and FAP, and a narrower, longer and spindle-shaped morphology of the cells was observed in the induced CAFs, which was similar to that commonly exhibited in activated fibroblasts; one of the characteristic features in CAFs (Fig. 1C). Taken together, these results confirmed the transformation of the HIFs into a CAF phenotype. Lactate assays revealed that the extracellular lactate excretion of CAFs was higher compared with that of HIFs after 12 and $24 \mathrm{~h}$ of culture (Fig. 1D). Correspondingly, the $\mathrm{pH}$ value of the medium was lower in CAFs after $24 \mathrm{~h}$ of culture (Fig. 1E). RT-qPCR and western blotting experiments demonstrated that the expression of PFK, a critical rate-limiting enzyme of glycolysis, and MCT4, an important lactate efflux transporter on the cell membrane, were significantly upregulated in the CAFs (Fig. 1F-H). These findings demonstrated the enhanced aerobic glycolytic metabolism of the CAFs.

CAFs promote the malignant behavior of CRC cells in vitro. To examine the effects of CAFs on the cancerous properties of the CRC cells, CM was collected from the CAFs to treat the cancer cells. Subsequently, CCK-8 proliferation assays demonstrated that CAFs were able to support cell proliferation of the HCT-116 and HT-29 cells compared with HIFs (Fig. 2A). Furthermore, compared with HIFs, CAFs significantly promoted the migration and invasion of HCT-116 and HT-29 cells, as shown in the wound healing assays (Fig. 2B and C) and Transwell migration and invasion assays (Fig. 2D).

EGCG directly inhibits the proliferation and migration of CRC cells in vitro. To examine the direct effects of EGCG on tumor cells, 25, 50 or $100 \mu \mathrm{M}$ EGCG was used to treat the HCT-116 and HT-29 cells. Proliferation assays demonstrated that EGCG could inhibit the proliferation of the CRC cells in a dose-dependent manner (Fig. 3A). The wound healing assay experiments indicated that treatment with EGCG elicited a direct suppressive effect on the migration of HCT-116 and HT-29 cells (Fig. 3B).

EGCG treatment suppresses the tumor-promoting capabilities of CAFs. Subsequently, the effects of EGCG on the malignancy-promoting abilities of CAFs were investigated. EGCG at concentrations of $0,25,50$ and $100 \mu \mathrm{M}$ was used to treat CAFs for $24 \mathrm{~h}$. After washing with PBS, EGCG-pretreated CAFs were cultured in serum-free fresh DMEM for a further $48 \mathrm{~h}$ to generate CM. Subsequently, CM from these EGCG-pretreated CAFs was collected for the further treatment of HCT-116 and HT-29 cells. Proliferation assays demonstrated that after pretreatment with EGCG, the capability of CAFs to stimulate cancer cell proliferation was decreased (Fig. 3C).

The direct effects of EGCG on the survival ability of HIFs was also investigated. However, treatment with EGCG at concentrations up to $100 \mu \mathrm{M}$ for $24 \mathrm{~h}$ did not produce any significant effects on the proliferation of HIFs (Fig. 3D). Therefore, it was concluded that the safe EGCG treatment conditions for CAFs were administration of a concentration of $50 \mu \mathrm{M}$ and a time of $24 \mathrm{~h}$. As shown in Fig. 3E-H, the ability of CAFs to promote the migration and invasion of HCT-116 and HT-29 cells was also suppressed following EGCG treatment in this condition.

EGCG treatment inhibits the aerobic glycolytic activity of $C A F s$. Previous studies have shown that EGCG is able to inhibit aerobic glycolysis in tumor cells $(14,15)$, and therefore it was possible to speculate that EGCG may exert an equivalent effect in CAFs. EGCG treatment decreased the lactate production of CAFs (Fig. 4A), as well as the expression of PFK and MCT4 (Fig. 4B-D), which indicated that the aerobic glycolytic activity of CAFs was inhibited following treatment with EGCG.

Lactate supplied by CAFs exerts an important role in tumor-stroma metabolic coupling. To further explore the metabolic coupling between CAFs and cancer cells, bindarit (an MCT4 inhibitor) was used to block the lactate supply from the CAFs. Bindarit treatment decreased the lactate concentration in CM after $6 \mathrm{~h}$ of culture of the CAFs (Fig. 4E). Although bindarit pretreatment of CAFs did not reveal any obvious effects on cancer cell proliferation (data not shown), the ability of CAFs to promote the migration and invasion of HCT-116 and HT-29 cells was reduced following bindarit treatment (Fig. 4F and G). Furthermore, the direct effects of bindarit on CRC cells were also examined. As shown in Fig. S1A and B, treatment with $100 \mu \mathrm{M}$ bindarit did not reveal any significant effects on the proliferation and migration of HCT-116 and HT-29 cells. Furthermore, siRNA experiments were performed to silence MCT4 expression in the CAFs to confirm its important role in the metabolic coupling process. The RT-qPCR and western blotting results confirmed the efficiency of MCT4 silencing in CAFs (Fig. 5A and B). As observed with the bindarit treatment, the tumor-promoting effects of CAFs were reduced following MCT4 silencing (Fig. 5C-E). Taken together, the findings in the present study demonstrated that EGCG exerted an anti-tumor role in impeding metabolic coupling between the CAFs and CRC cells (Fig. 5F). 
A

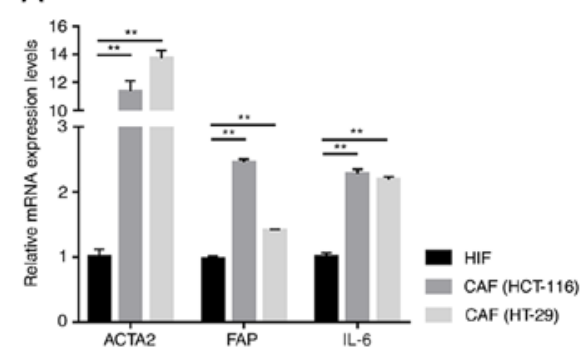

C

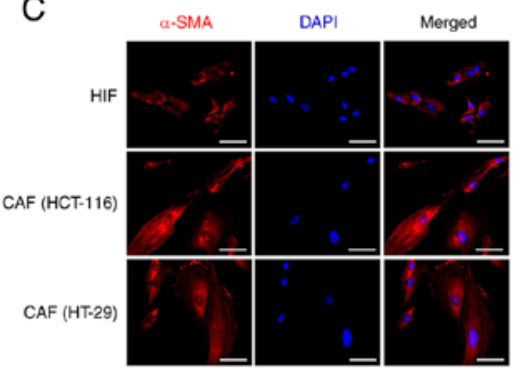

D

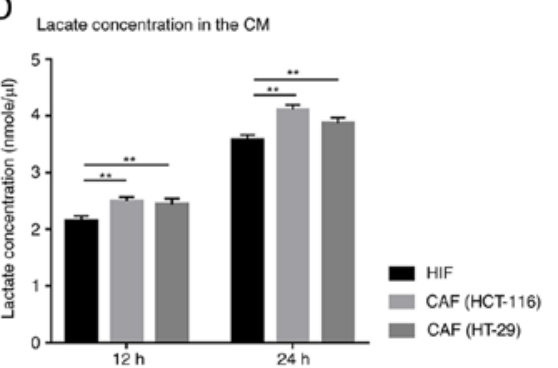

$\mathrm{F}$

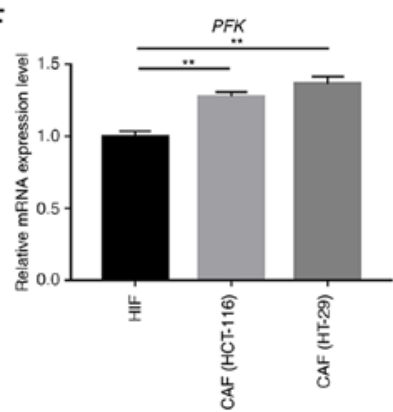

B
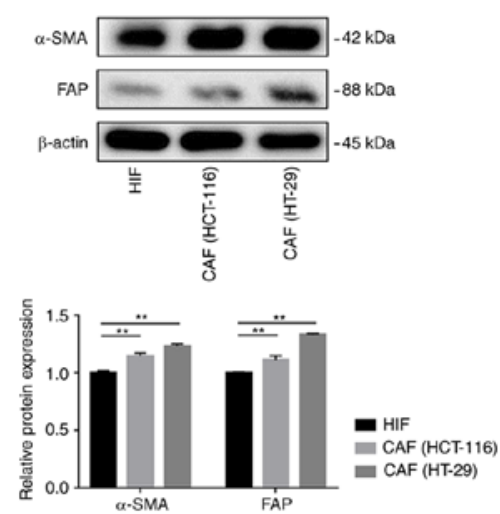

FAP

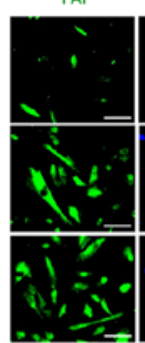

DAPI

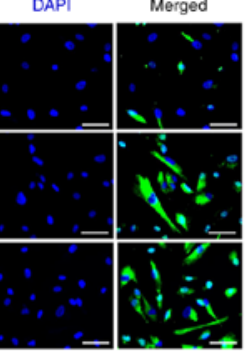

E

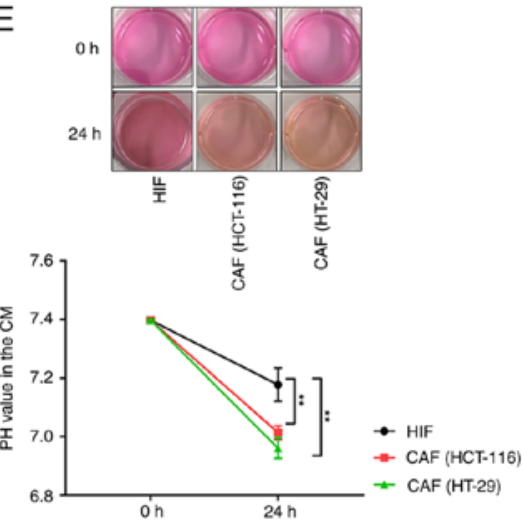

$\mathrm{G}$

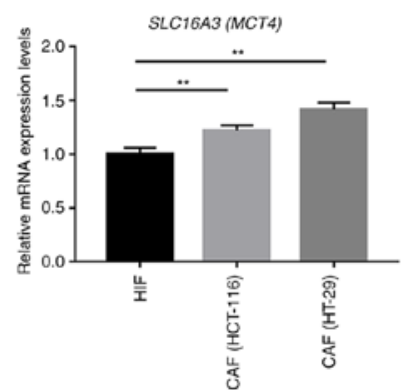

$\mathrm{H}$
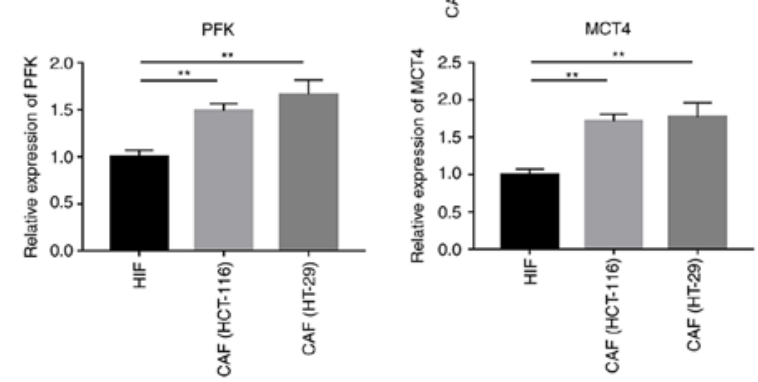

Figure 1. Fibroblasts co-cultured with tumor cells exhibit a CAF phenotype with enhanced aerobic glycolysis. (A) After co-culture with HCT-116 or HT-29 CRC cells, the gene expression levels of CAF markers were detected in HIFs by RT-qPCR analysis. (B) The expression levels of $\alpha$-SMA and FAP of HIFs and CAFs were measured by western blot analysis. (C) Immunofluorescence staining results, showing the expression levels of $\alpha$-SMA and FAP in HIFs and CAFs (scale bar, $50 \mu \mathrm{m}$ ). (D) Extracellular lactate excretion rates of HIFs and CAFs were evaluated according to the lactate concentration of CM in lactate assays. (E) Typical images and $\mathrm{pH}$ values of CM in HIF and CAF groups. Gene expression levels of (F) PFK and (G) MCT4 were detected in HIFs and CAFs by RT-qPCR analysis. (H) Western blotting results also confirmed the overexpression of PFK and MCT4 in CAFs compared with HIFs. ** P<0.01. $\alpha$-SMA, $\alpha$-smooth muscle actin; ACTA2, actin $\alpha$-2; FAP, fibroblast activation protein; HIF, human intestinal fibroblast; CAFs, cancer-associated fibroblasts; CM, conditioned medium; RT-qPCR, reverse transcription-quantitative PCR; PFK, phosphofructokinase; MCT4, monocarboxylate transporter 4. 
A

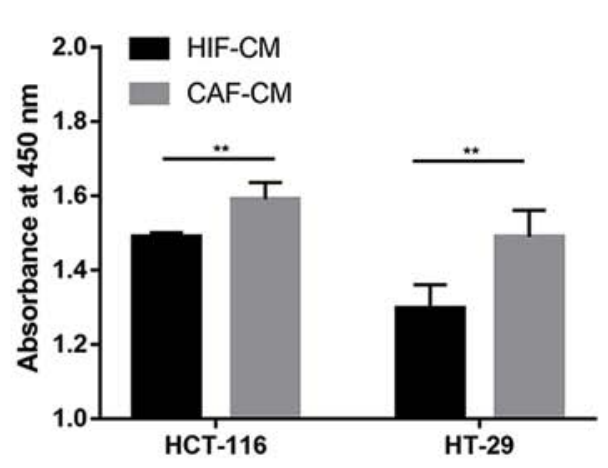

B

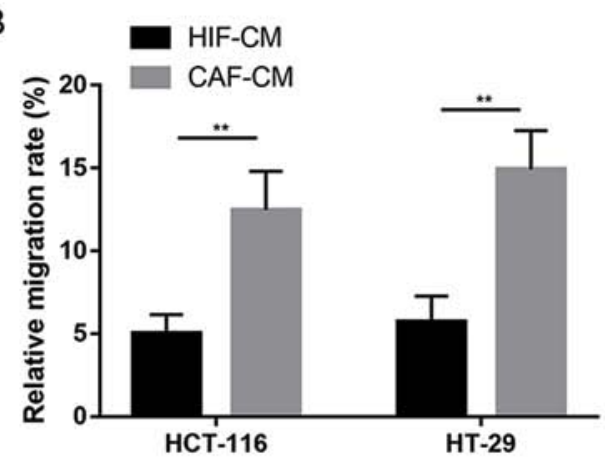

C
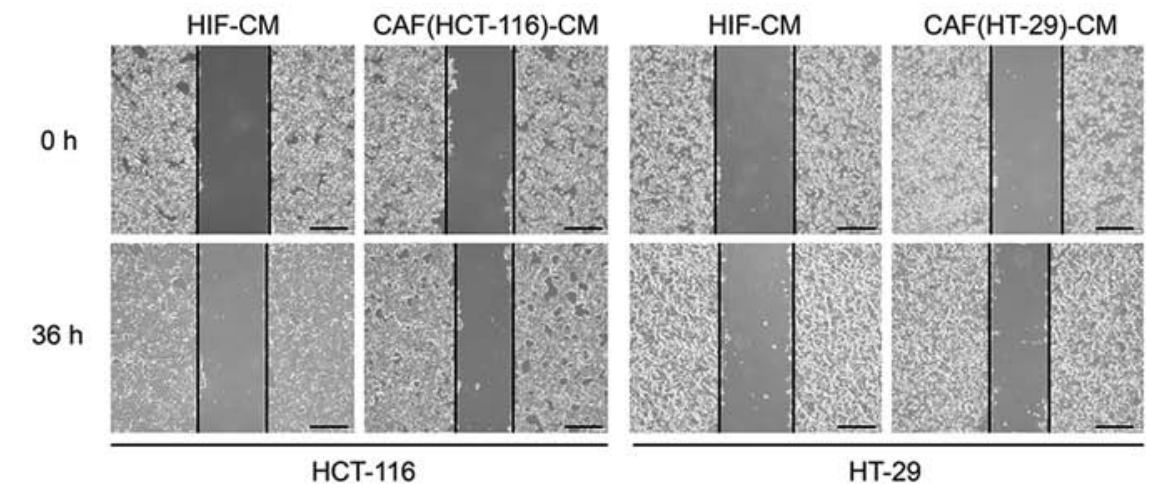

HT-29
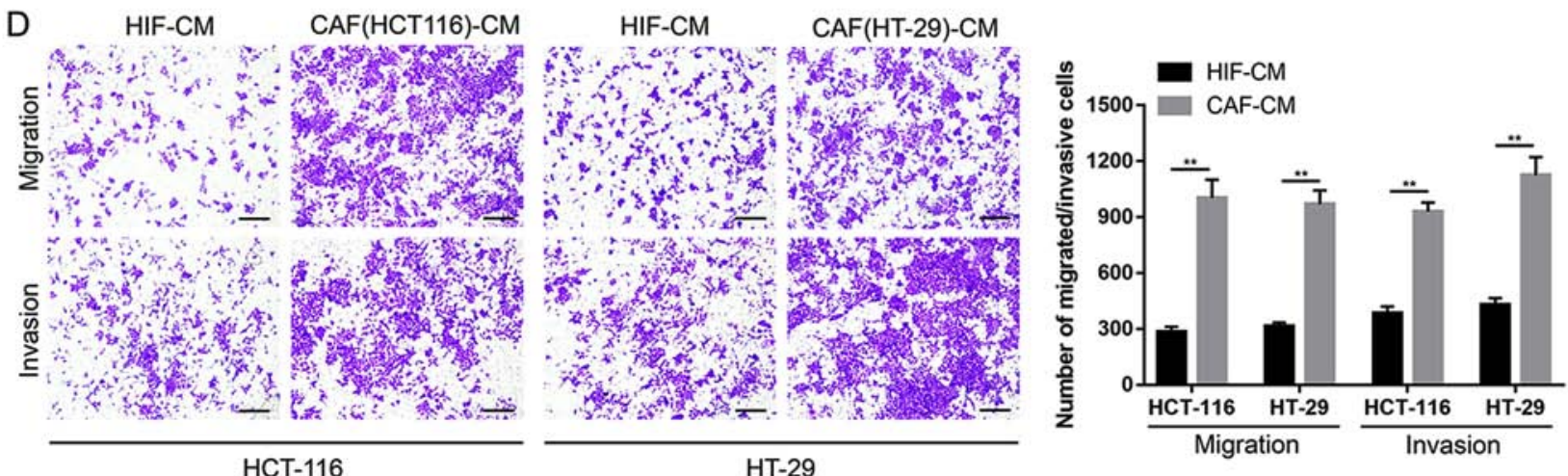

Figure 2. CAFs promote the malignant behavior of CRC cells in vitro. (A) Cell Counting Kit-8 assay results demonstrated the promoting effect of CAFs on the proliferation of HCT-116 and HT-29 cells after $48 \mathrm{~h}$ culture. (B and C) Wound healing (scale bar, $400 \mu \mathrm{m}$ ) and (D) Transwell migration and invasion assays (scale bar, $200 \mu \mathrm{m}$ ) indicated that CAFs promoted HCT-116 and HT-29 cell migration and invasion compared with HIFs. ${ }^{* *} \mathrm{P}<0.01$. HIFs, human intestinal fibroblasts; CAFs, cancer-associated fibroblasts; CRC, colorectal cancer; CM, conditioned medium.

\section{Discussion}

In the present study, it was demonstrated that CAFs showed enhanced aerobic glycolytic activity, and were able to promote CRC progression through lactate secretion compared with normal fibroblasts. Both EGCG treatment and MCT4 inhibition were shown to reduce the aerobic glycolytic activity of CAFs, thereby impeding their metabolic coupling with CRC cells and reducing their promoting effects on tumor progression. The therapeutic application of EGCG for targeting glucose metabolism in stromal cells in the TME was therefore been shown to be a safe and effective strategy as an alternative means of anti-cancer therapy.

As an important stromal cell type in the TME, CAFs are able to promote tumor progression through various direct or indirect effects, including paracrine secretion of cytokines $(26,27)$. Recent research on metabolic heterogeneity has shifted its focus from cancer cells to the reprogramming of tumor-stroma metabolic coupling, which results from the profound crosstalk between CAFs and cancer cells $(4,5,9,12,28)$. This new paradigm has been termed 'two-compartment tumor metabolism' and the 'reverse Warburg effect' $(6,12,29-33)$, and its existence has been confirmed in lung cancer (34), pancreatic cancer (35), oral squamous cell carcinoma $(36,37)$, breast cancer $(7,38)$, prostate cancer (39) and nasopharyngeal cancer (40). In terms of the specific details, CAFs undergo metabolic reprogramming, which manifests itself as enhanced aerobic glycolysis. High amounts of lactic acid secreted by CAFs with an upregulated expression of MCT4 as an energy substrate are absorbed by cancer cells, and these participate in the metabolic alteration of cancer cells, thereby enhancing 

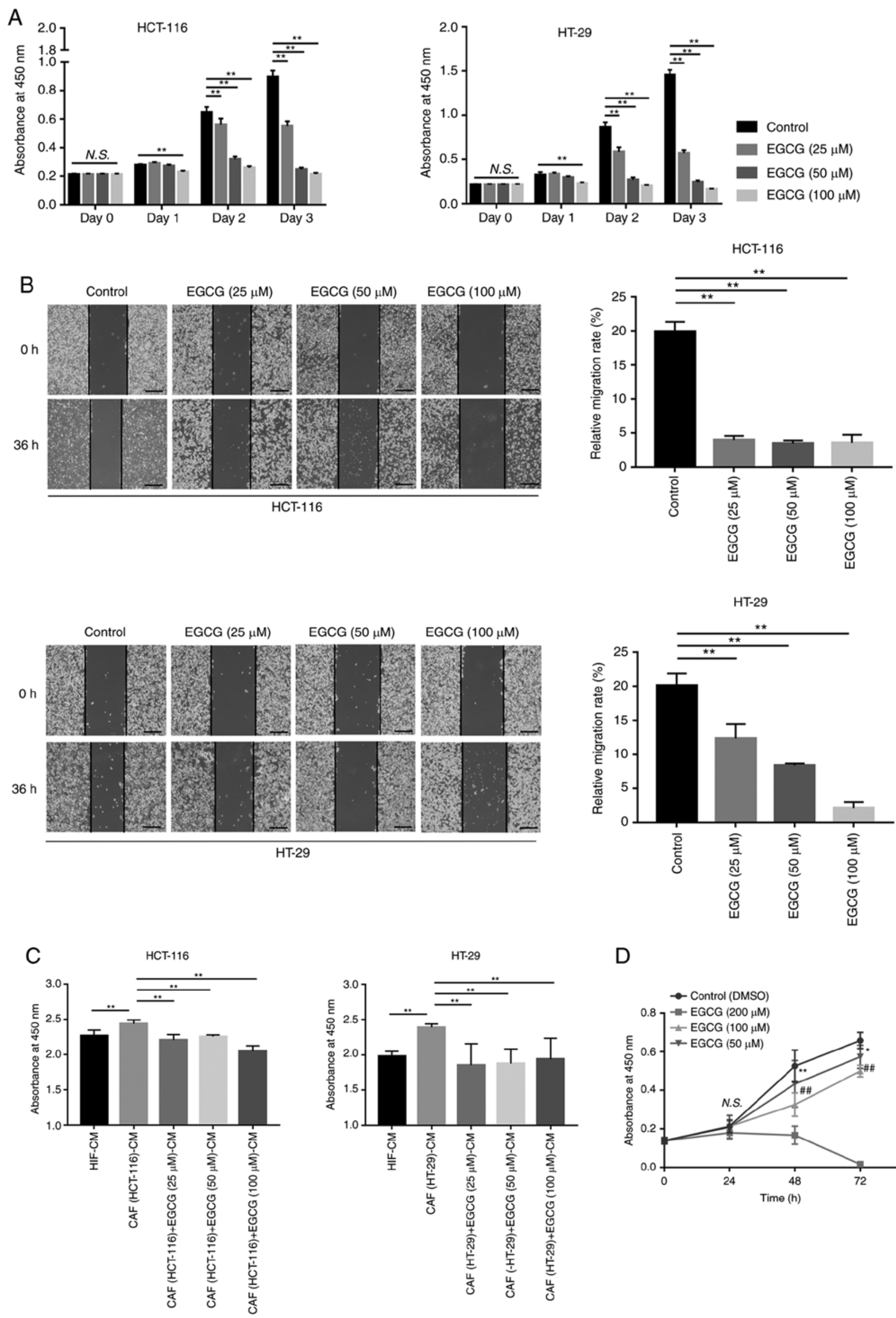

D

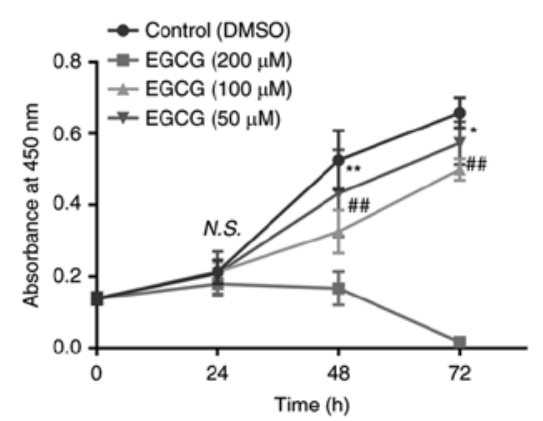

Figure 3. Continued. 


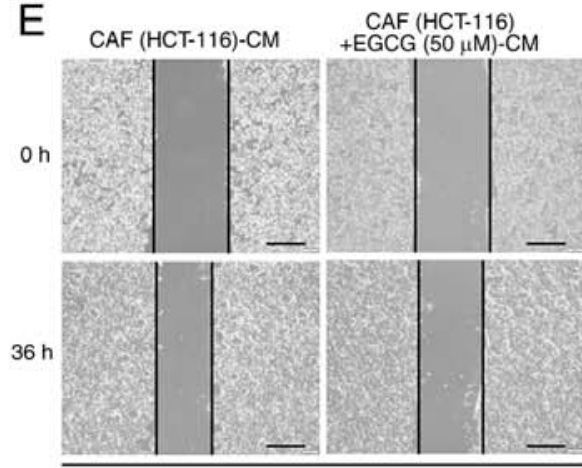

HCT-116

F

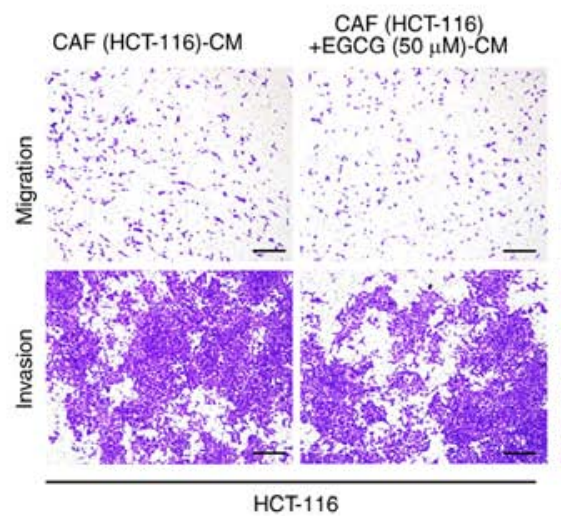

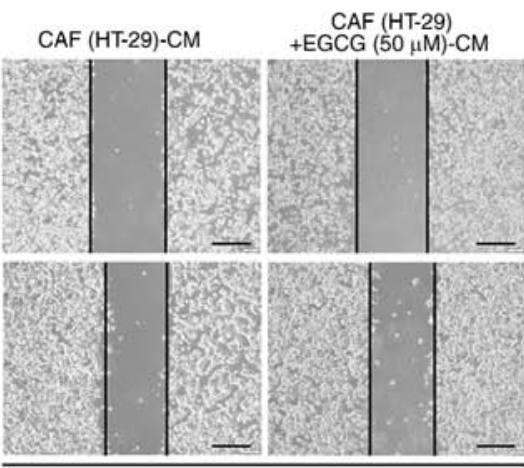

HT-29

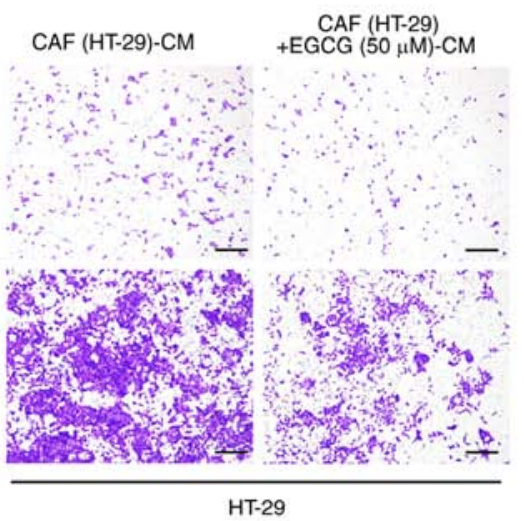

CAF-CM

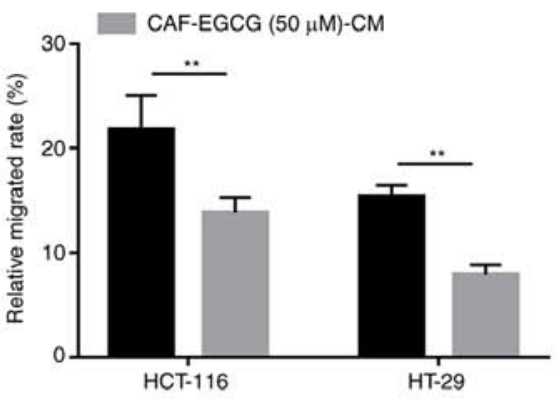

G

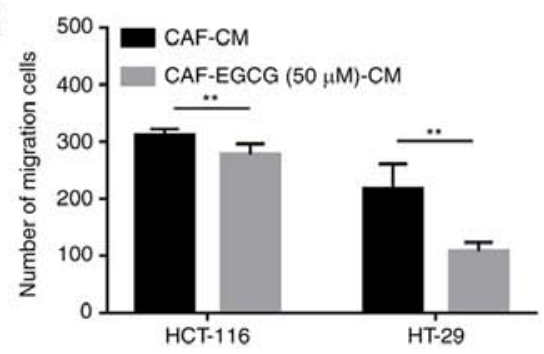

$\mathrm{H}$

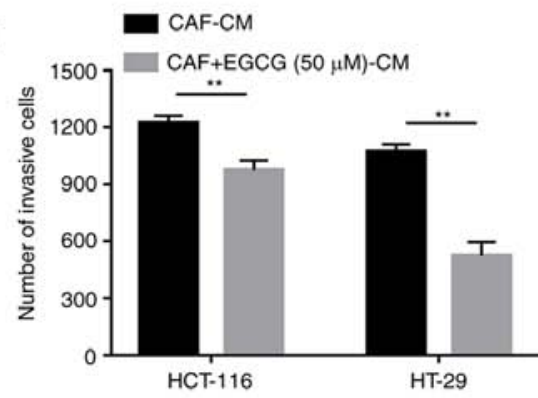

Figure 3. The effects of EGCG treatment on CRC cells and CAFs. (A) Cell Counting Kit-8 assay results demonstrated that treatment with 25,50 and $100 \mu \mathrm{M}$ EGCG directly suppressed the proliferation of HCT-116 and HT-29 cells after 1-3 days of culture. (B) Wound healing (scale bar, $400 \mu \mathrm{m}$ ) indicated the direct effects of different concentrations of EGCG on the migration of HCT-116 and HT-29 cells. (C) Cell Counting Kit-8 results demonstrated that treatment with 25, 50 and $100 \mu \mathrm{M}$ EGCG suppressed the promoting effect of CAFs on the proliferation of HCT-116 and HT-29 cells after $48 \mathrm{~h}$ of culture. (D) Cell Counting Kit- 8 assay results showed the direct effects of different concentrations and durations of EGCG treatment on the proliferation of HIFs. "P<0.05 and ${ }^{* *} \mathrm{P}<0.01$ vs. $50 \mu \mathrm{M}$ EGCG; ${ }^{\# \#} \mathrm{P}<0.01$ vs. $100 \mu \mathrm{M}$ EGCG. (E) Wound healing (scale bar, $400 \mu \mathrm{m}$ ) and (F-H) Transwell migration and invasion assays (scale bar, $200 \mu \mathrm{m}$ ) indicated that EGCG treatment suppressed the promoting effect of CAFs on the migration and invasion of HCT-116 and HT-29 cells. "* P<0.01. HIFs, human intestinal fibroblasts; EGCG, epigallocatechin-3-gallate; CAFs, cancer-associated fibroblasts; CRC, colorectal cancer; $\mathrm{CM}$, conditioned medium. N.S., not significant.

tumor malignancy. In the present study, the western blotting results showed that the expression levels of $\alpha$-SMA and FAP, the two most widely used markers for identifying CAFs $(41,42)$, were upregulated in HIFs after co-culture. Moreover, the results from the immunofluorescence staining experiments confirmed the upregulation of $\alpha$-SMA and FAP, and a narrower, longer and spindle-shaped morphology of the cells was observed in the induced CAFs that was similar to that commonly exhibited in activated fibroblasts, as one of their characteristic features in CAFs (43). The current consensus is that CAFs are a stromal cell population with cell-of-origin, phenotypic and functional heterogeneity (43). Although the exact method of identifying CAFs is still controversial, it is generally hypothesized that CAFs are derived from activated myofibroblasts in the TME. The typical spindle-shaped morphology changes compared with quiescent fibroblasts, and the upregulation of several specific CAF markers that are widely used, such as $\alpha$-SMA, FAP and IL-6, can be used to identify CAFs (41-43). Recently, co-culture with cancer cells has become a way to obtain and study CAFs in vitro $(44,45)$. Thus, it was concluded that HIFs transformed into a CAF phenotype. The induced CAFs exhibited an enhanced rate of aerobic glycolysis, which was characterized by upregulation of the glycolytic rate-limiting enzyme PFK and lactate transporter MCT4, as well as increased lactate production. Moreover, CM from CAFs had a marked ability to promote the malignancy of CRC cells in vitro.

EGCG is a safe natural substance, and its direct anti-tumor properties have been extensively studied $(15,20,46)$. Consistent with a previous study (17), the results of the present study also revealed that EGCG was able to directly inhibit the proliferation and migration of CRC cells. Moreover, it has been shown for the first time, to the best of our knowledge, that treatment with EGCG at a concentration of $50 \mu \mathrm{M}$ (an assured safe dose) for $24 \mathrm{~h}$ led to a reduction in the aerobic glycolytic capacity of 
A

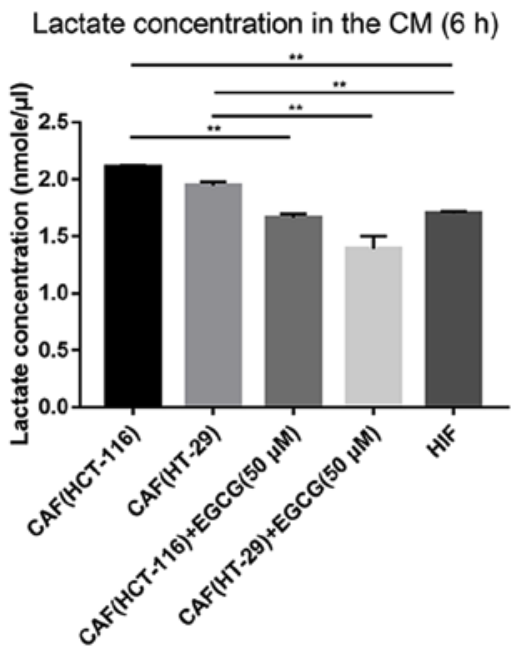

B

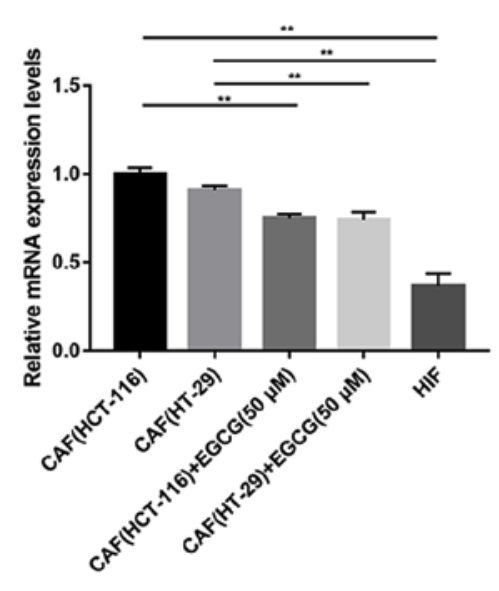

C

$S L C 16 A 3(M C T 4)$

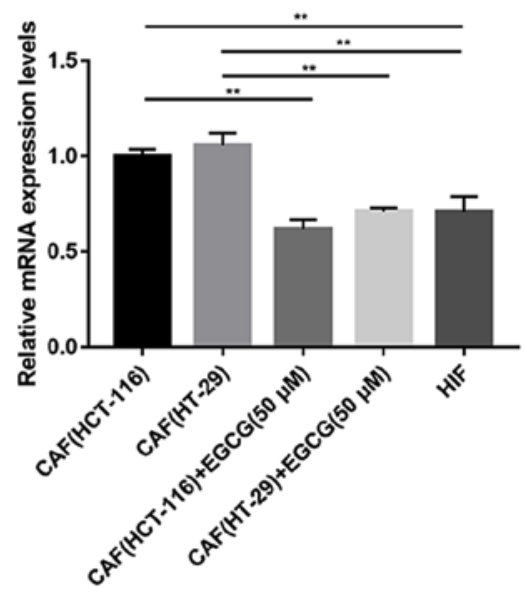

D
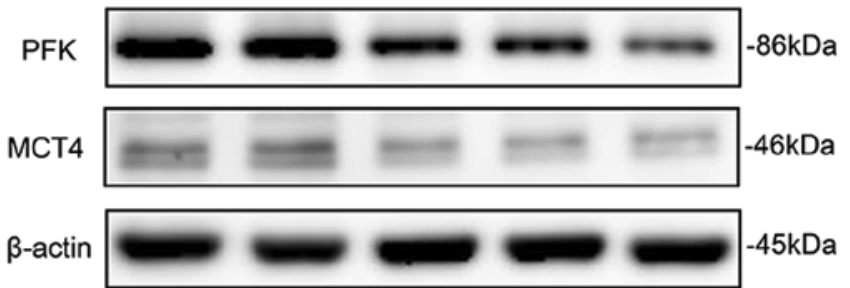

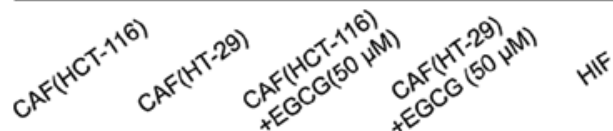
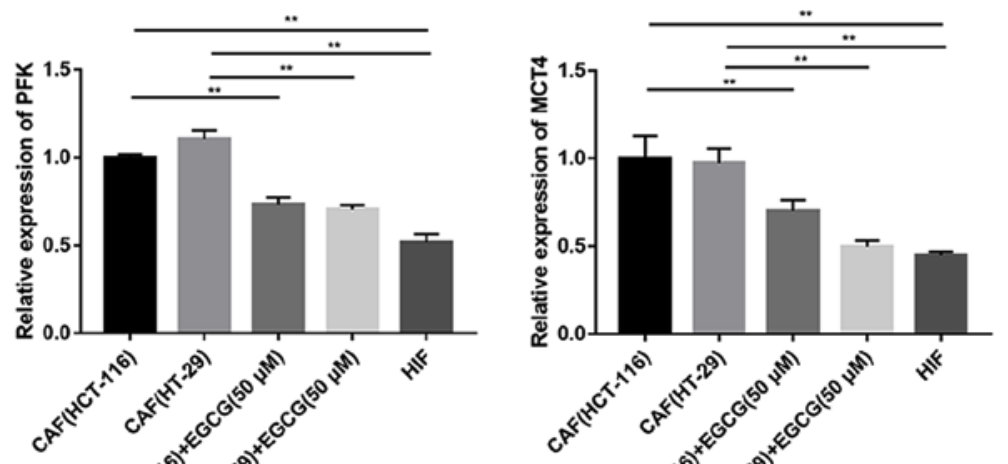

E

Lactate concentration in the $\mathrm{CM}(6 \mathrm{~h})$
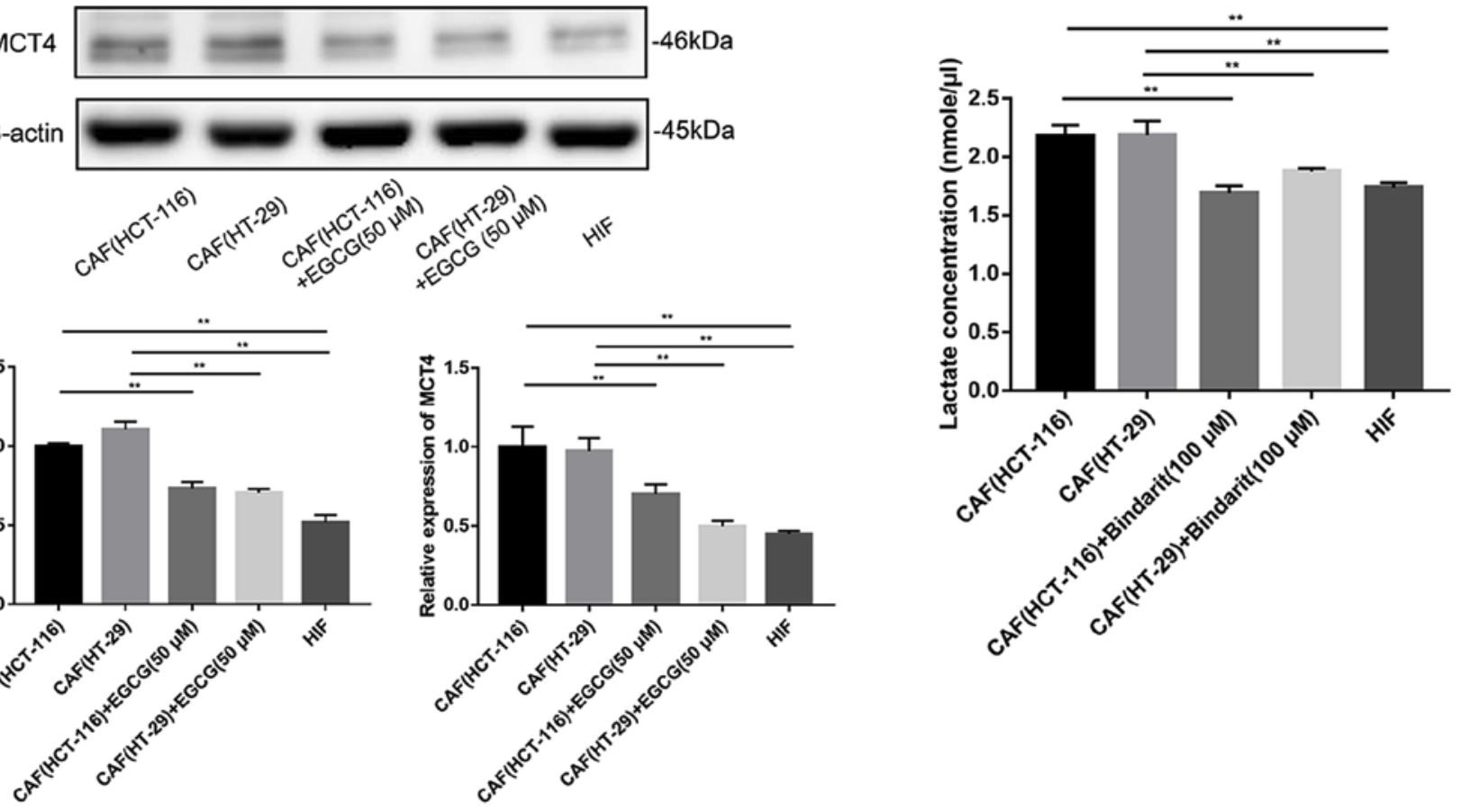

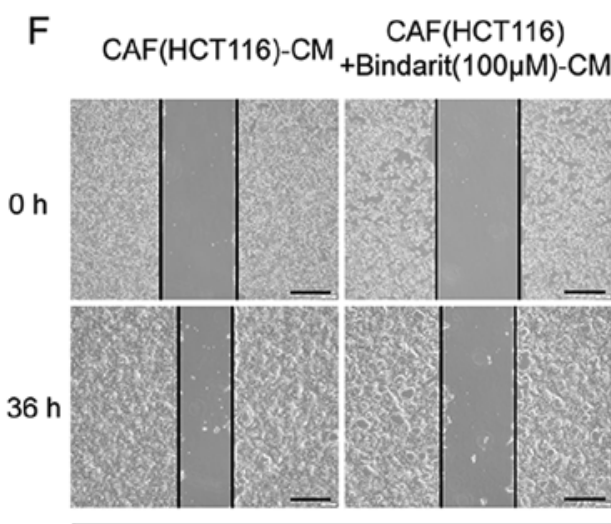

HCT-116

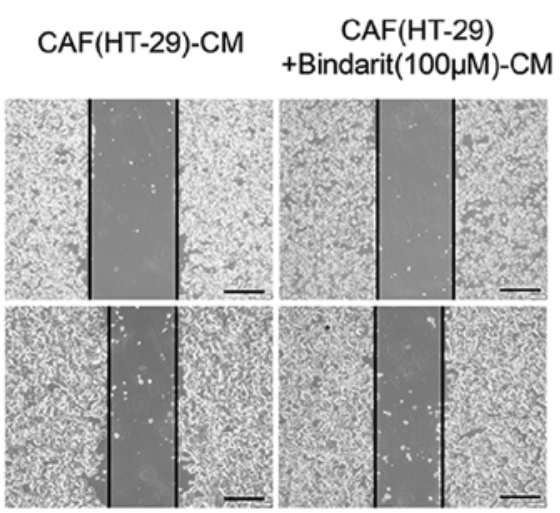

HT-29

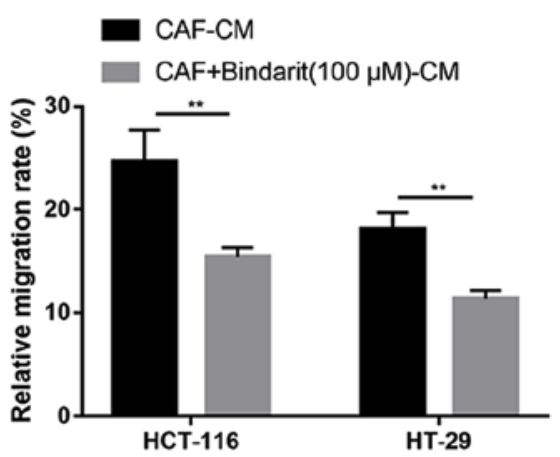

Figure 4. Continued. 
G

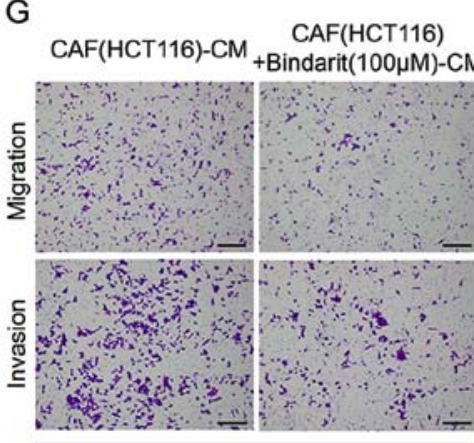

HCT-116
$\mathrm{CAF}(\mathrm{HT}-29)-\mathrm{CM}$

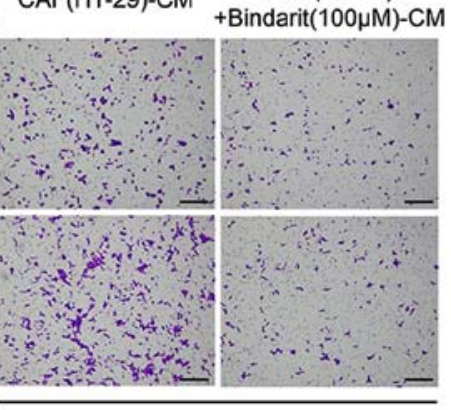

HT-29

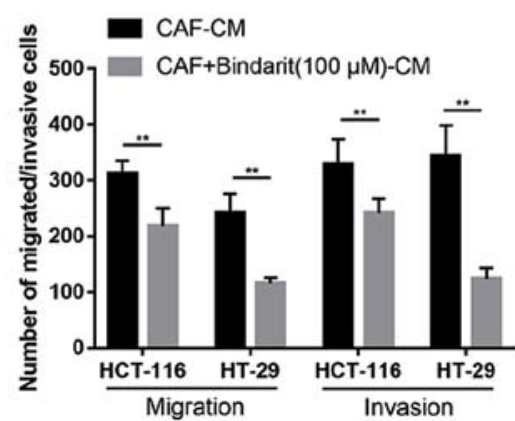

Figure 4. EGCG treatment inhibits the aerobic glycolytic activity in CAFs to suppress tumor-stroma metabolic coupling. (A) Lactate assay results showed EGCG treatment decreased the lactate production of CAFs in CM after $6 \mathrm{~h}$ of culture. (B) Gene expression levels of (B) PFK and (C) MCT4 were detected in HIFs, CAFs and EGCG-treated CAFs by RT-qPCR analysis. (D) Western blotting results showed the protein expression levels of PFK and MCT4 amongst these groups. (E) Lactate assays were used to analyze lactate production in CAFs treated with bindarit. (F) Wound healing (scale bar, $400 \mu \mathrm{m})$ and (G) Transwell migration and invasion assays (scale bar, $200 \mu \mathrm{m}$ ) indicated that inhibition of MCT4 in CAFs suppressed the promoting effect of CAFs on the migration and invasion of HCT-116 and HT-29 cells. ${ }^{* *} \mathrm{P}<0.01$. HIFs, human intestinal fibroblasts; EGCG, epigallocatechin-3-gallate; CAFs, cancer-associated fibroblasts; CM, conditioned medium; PFK, phosphofructokinase; MCT4, monocarboxylate transporter 4.

CAFs. Specifically, PFK and MCT4 were downregulated, and the efflux of lactic acid was reduced upon administering this dose of EGCG. Interestingly, EGCG-treated CAFs exhibited a reduced ability to promote $\mathrm{CRC}$ malignancy. These findings have expanded the concept of the anti-tumor mechanism of EGCG, suggesting that it may be used as a safe anti-cancer adjuvant targeting the TME.

According to the 'two-compartment' metabolic coupling hypothesis, MCT4 is upregulated in CAFs and contributes to the excretion of lactic acid, while MCT1 is overexpressed in cancer to increase lactate uptake. Through this lactic acid shuttle from CAFs to cancer, lactate becomes an energy source for further utilization by cancer cells $(6,9,28,47,48)$. From this perspective, targeting of the MCT family and use of MCT1/MCT4 inhibitors to disrupt the 'lactic acid shuttle' for the 'reverse Warburg effect' has become an anti-tumor strategy $(8,10)$. In fact, silencing of MCT4 in CAFs weakens their ability to secrete lactic acid and its subsequent promoting effect on the invasion of breast cancer (49). Futagi et al (50) demonstrated that bindarit acts as the first potent and highly selective inhibitor of human MCT4, with a $K_{\mathrm{i}}$ value of $30.2 \pm 1.4 \mu \mathrm{M}$. In the present study, bindarit was used at a concentration of $100 \mu \mathrm{M}$, which is sufficient for the inhibition of MCT4. Indeed, using various or more specific inhibitors of MCT4 may more convincingly confirm this conclusion. However, novel selective MCT4 inhibitors remain to be identified/designed. Thus, siRNA technology was also to specifically silence MCT4 in CAFs in the present study to provide more experimental support for the conclusion drawn. Similar to the experiments with EGCG, using an MCT4 inhibitor or silencing MCT4 expression also weakened the tumor-promoting effects of CAF. These experiments further confirmed that the suppressive effects of EGCG on CAFs may be attributed to blocking aerobic glycolysis.

Although novel findings have been presented in the present study, further work is required. Firstly, the lack of further evidence demonstrating transformation of HIFs into a CAF-like phenotype is a limitation of the present study. Secondly, for silencing MCT4, siRNA technology was used, the absence of shRNA experiments is another limitation of this study. Additionally, the detailed mechanism through which lactic acid stimulated tumor progression is still not fully understood. The absorbed lactic acid could serve as the main source of carbon in tumors and enter the tricarboxylic acid cycle (51). It could also directly promote the epithelial-mesenchymal transition and the expression of angiogenesis-associated genes in cancer (52). Therefore, in the context of nutrient metabolism, lactate should be studied further as a signaling molecule. Furthermore, another study demonstrated that CAFs were able to directly transfer mitochondria to tumor cells in order to enhance their oxyphosphate phosphorylation (38). This phenomenon may explain why the MCT4 inhibitor did not affect the proliferation of cancer cells. Metabolic heterogeneity in the TME and the dynamics of metabolic coupling both require further investigation.

In conclusion, the present study revealed the metabolic coupling of cancer cells and CAFs in CRC, with the resultant effects on tumor progression. Knowledge of the anti-tumor mechanism of EGCG has also been expanded through targeting of the glycolysis of CAFs in the TME. Collectively, the findings of the present study should prove to be valuable for further clarifying tumor-stroma metabolic coupling and proposing novel therapeutic strategies.

\section{Acknowledgements}

Not applicable.

\section{Funding}

This work was supported by the Grants-in-Aid for Scientific Research (grant nos. 20K08957 to YM), Taiho Pharmaceutical Co., Ltd. and Tsumura Pharmaceutical Co., Ltd.

\section{Availability of data and materials}

The datasets used and/or analyzed during the current study are available from the corresponding author on reasonable request. 


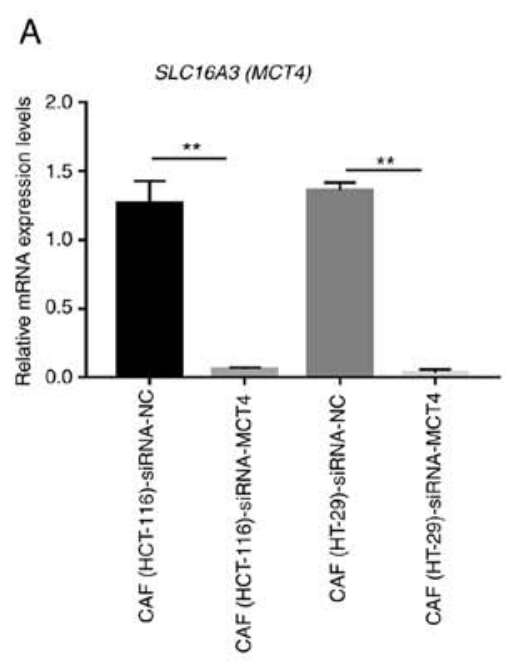

B
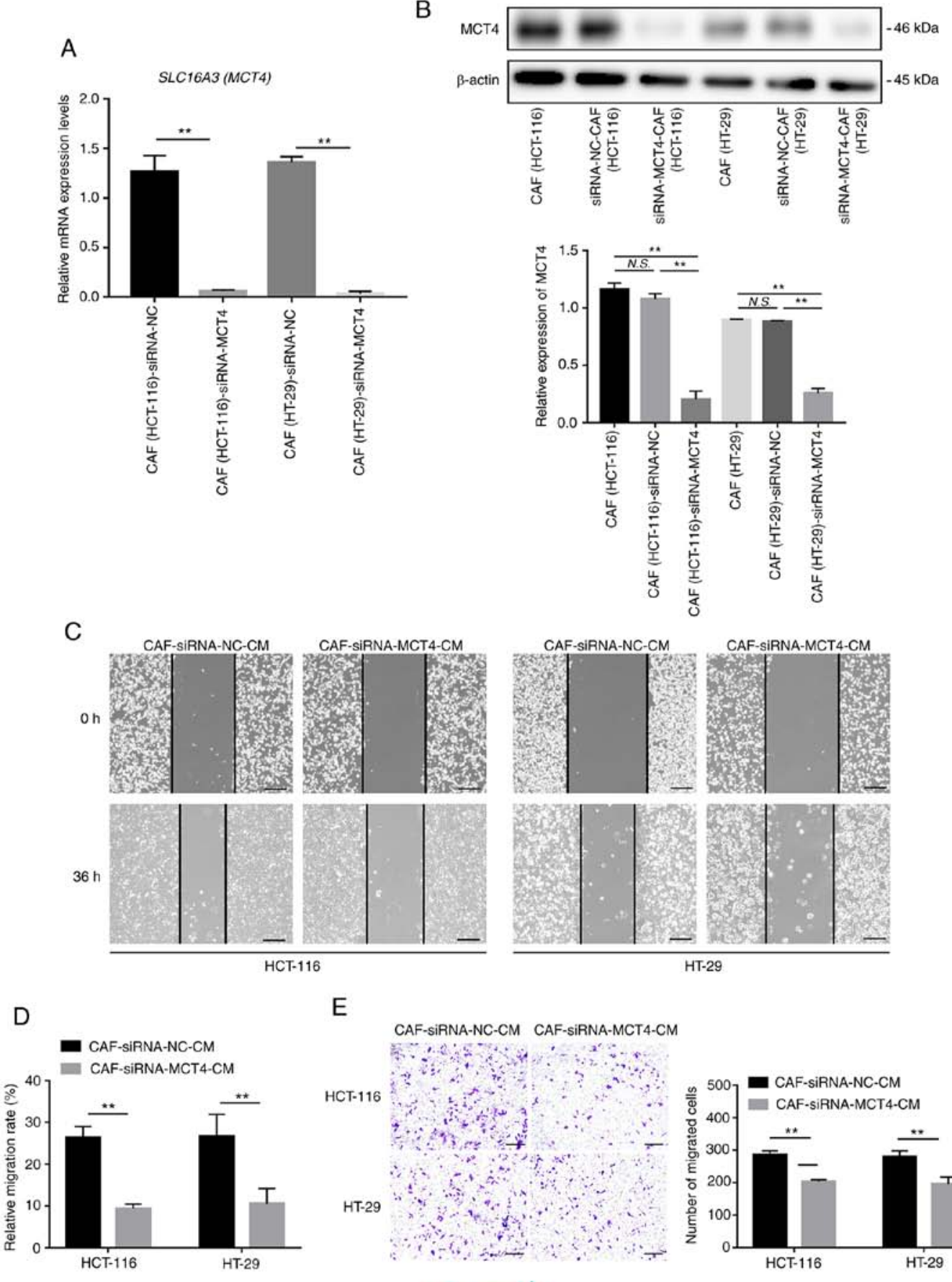

E
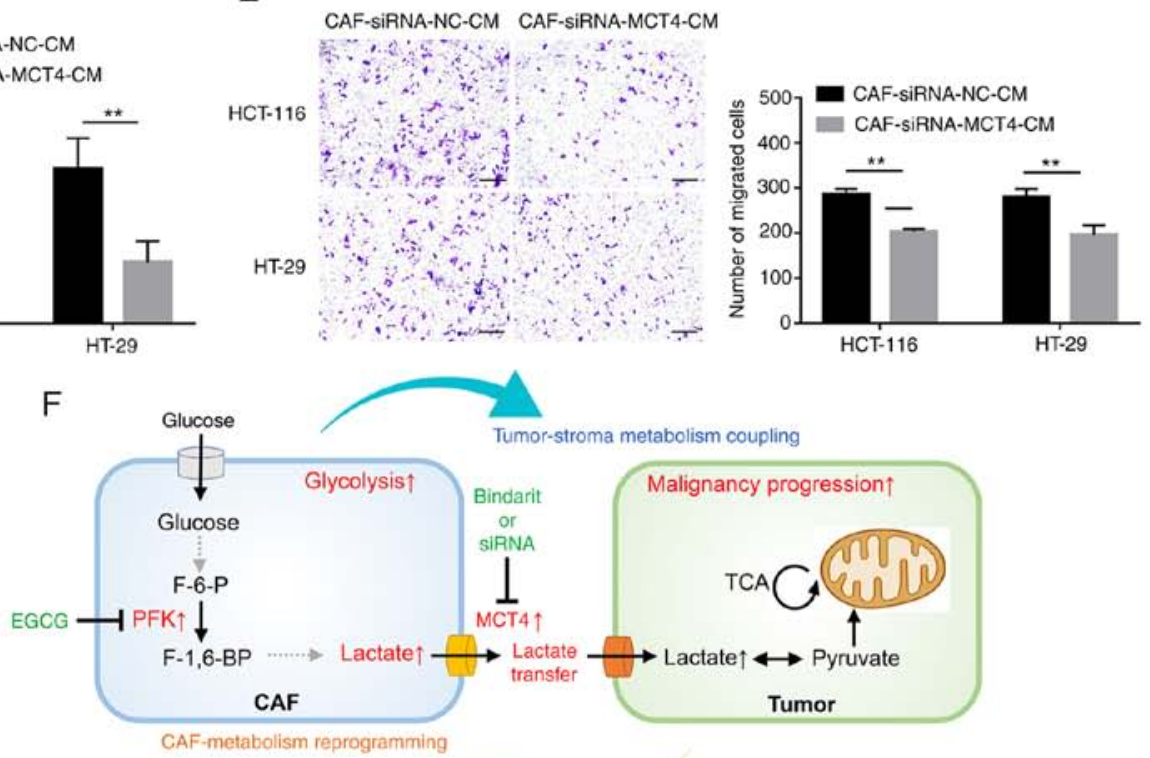

Figure 5. MCT4 silencing in CAFs suppressed its tumor-promoting effects. (A) Gene expression levels of MCT4 were detected in siRNA-treated CAFs by RT-qPCR analysis. (B) Western blotting results confirmed the downregulation of MCT4 protein levels in CAFs after siRNA treatment. (C and D) Wound healing (scale bar, $400 \mu \mathrm{m}$ ) and (E) Transwell migration assays (scale bar, $200 \mu \mathrm{m}$ ) indicated that knockdown of MCT4 in CAFs suppressed the promoting effect of CAFs on the migration and invasion of HCT-116 and HT-29 cells. (F) Schematic representation of EGCG inhibition of the metabolic coupling between CRC and CAFs via targeting of aerobic glycolysis in CAFs. ${ }^{* *} \mathrm{P}<0.01$. HIFs, human intestinal fibroblasts; EGCG, epigallocatechin-3-gallate; CAFs, cancer-associated fibroblasts; CM, conditioned medium; F-6-P, fructose-6-phosphate; F-1,6-BP, fructose-1,6-bisphosphate; PFK, phosphofructokinase; MCT4, monocarboxylate transporter 4; tricarboxylic acid cycle, TCA cycle; NC, negative control; N.S., not significant. 


\section{Authors' contributions}

SC, YM and MS designed the experiments. SC, TT, HK and CT performed the experiments and collected the data. YM, SY and CT analyzed and interpreted the data. SHC and YW drafted the manuscript. MN, YM and MS revised the paper critically for important intellectual content. SHC and MN confirmed the authenticity of all the raw data. SHC and MN agree to be accountable for all aspects of the work in ensuring that questions related to the accuracy or integrity of any part of the work are appropriately investigated and resolved. All the authors have read and approved the final version of the manuscript for publication.

\section{Ethics approval and consent to participate}

This study was approved by The Ethics Committee of Tokushima University Hospital (TOCMS: 2901-2). This study strictly followed the ethical guidance of Ministry of Health, Labour and Welfare in Japan and Tokushima University regulations.

\section{Patient consent for publication}

Not applicable.

\section{Competing interests}

The conflicts of interest with regard to our study are as follows: grant support from Taiho Pharmaceutical Co., Ltd, and Tsumura Co., Ltd.

\section{References}

1. Bray F, Ferlay J, Soerjomataram I, Siegel RL, Torre LA and Jemal A: Global cancer statistics 2018: GLOBOCAN estimates of incidence and mortality worldwide for 36 cancers in 185 countries. CA Cancer J Clin 68: 394-424, 2018.

2. Siegel RL, Miller KD, Goding Sauer A, Fedewa SA, Butterly LF, Anderson JC, Cercek A, Smith RA and Jemal A: Colorectal Cancer statistics, 2020. CA Cancer J Clin 70: 145-164, 2020.

3. Hanahan D and Weinberg RA: Hallmarks of cancer: The next generation. Cell 144: 646-674, 2011.

4. Gentric $\mathrm{G}$ and Mechta-Grigoriou F: Tumor cells and cancer-associated fibroblasts: An updated metabolic perspective. Cancers (Basel) 13: 399, 2021.

5. Eisenberg L, Eisenberg-Bord M, Eisenberg-Lerner A and Sagi-Eisenberg R: Metabolic alterations in the tumor microenvironment and their role in oncogenesis. Cancer Lett 484: 65-71, 2020.

6. Pavlides S, Whitaker-Menezes D, Castello-Cros R, Flomenberg N, Witkiewicz AK, Frank PG, Casimiro MC, Wang C, Fortina P, Addya S, et al: The reverse Warburg effect: Aerobic glycolysis in cancer associated fibroblasts and the tumor stroma. Cell Cycle 8: 3984-4001, 2009.

7. Becker LM, O'Connell JT, Vo AP, Cain MP, Tampe D, Bizarro L, Sugimoto H, McGow AK, Asara JM, Lovisa S, et al: Epigenetic reprogramming of cancer-associated fibroblasts deregulates glucose metabolism and facilitates progression of breast cancer. Cell Rep 31: 107701, 2020.

8. Jung JG and Le A: Targeting metabolic cross talk between cancer cells and cancer-associated fibroblasts. Adv Exp Med Biol 1311: 205-214, 2021.

9. Martinez-Outschoorn UE, Lisanti MP and Sotgia F: Catabolic cancer-associated fibroblasts transfer energy and biomass to anabolic cancer cells, fueling tumor growth. Semin Cancer Biol 25: 47-60, 2014.

10. Fu Y, Liu S, Yin S, Niu W, Xiong W, Tan M, Li G and Zhou M: The reverse Warburg effect is likely to be an Achilles' heel of cancer that can be exploited for cancer therapy. Oncotarget 8: 57813-57825, 2017.
11. Martinez-Outschoorn UE, Peiris-Pagés M, Pestell RG, Sotgia F and Lisanti MP: Cancer metabolism: A therapeutic perspective. Nat Rev Clin Oncol 14: 11-31, 2017.

12. Benny S, Mishra R, Manojkumar MK and Aneesh TP: From Warburg effect to reverse Warburg effect; the new horizons of anti-cancer therapy. Med Hypotheses 144: 110216, 2020.

13. Keller F, Bruch R, Schneider R, Meier-Hubberten J, Hafner M and Rudolf R: A scaffold-free 3-D co-culture mimics the major features of the reverse Warburg effect in vitro. Cells 9: 1900, 2020.

14. Jankun J, Selman SH, Swiercz R and Skrzypczak-Jankun E: Why drinking green tea could prevent cancer. Nature 387: 561, 1997.

15. Aggarwal V, Tuli HS, Tania M, Srivastava S, Ritzer EE, Pandey A, Aggarwal D, Barwal TS, Jain A, Kaur G, et al: Molecular mechanisms of action of epigallocatechin gallate in cancer: Recent trends and advancement. Semin Cancer Biol. May 24, 2020. (Epub ahead of print). doi: 10.1016/j.semcancer.2020.05.011.

16. Shen X, Zhang Y, Feng Y, Zhang L, Li J, Xie YA and Luo X: Epigallocatechin-3-gallate inhibits cell growth, induces apoptosis and causes S phase arrest in hepatocellular carcinoma by suppressing the AKT pathway. Int J Oncol 44: 791-796, 2014.

17. Luo KW, Xia J, Cheng BH, Gao HC, Fu LW and Luo XL: Tea polyphenol EGCG inhibited colorectal-cancer-cell proliferation and migration via downregulation of STAT3. Gastroenterol Rep (Oxf) 9: 59-70, 2020.

18. Wei R, Mao L, Xu P, Zheng X, Hackman RM, Mackenzie GG and Wang Y: Suppressing glucose metabolism with epigallocatechin-3-gallate (EGCG) reduces breast cancer cell growth in preclinical models. Food Funct 9: 5682-5696, 2018.

19. Wang J, Xie Y, Feng Y, Zhang L, Huang X, Shen X and Luo X: (-)-Epigallocatechingallate induces apoptosis in B lymphoma cells via caspase-dependent pathway and Bcl-2 family protein modulation. Int J Oncol 46: 1507-1515, 2015.

20. Chow HH, Cai Y, Hakim IA, Crowell JA, Shahi F, Brooks CA, Dorr RT, Hara Y and Alberts DS: Pharmacokinetics and safety of green tea polyphenols after multiple-dose administration of epigallocatechin gallate and polyphenon $\mathrm{E}$ in healthy individuals. Clin Cancer Res 9: 3312-3319, 2003.

21. Kuo PL and Lin CC: Green tea constituent (-)-epigallocatechin-3-gallate inhibits Hep G2 cell proliferation and induces apoptosis through p53-dependent and Fas-mediated pathways. J Biomed Sci 10: 219-227, 2003.

22. Gupta S, Hastak K, Afaq F, Ahmad N and Mukhtar H: Essential role of caspases in epigallocatechin-3-gallate-mediated inhibition of nuclear factor kappa B and induction of apoptosis. Oncogene 23: 2507-2522, 2004.

23. Li S, Xia Y, Chen K, Li J, Liu T, Wang F, Lu J, Zhou Y and Guo C: Epigallocatechin-3-gallate attenuates apoptosis and autophagy in concanavalin A-induced hepatitis by inhibiting BNIP3. Drug Des Devel Ther 10: 631-647, 2016.

24. Li S, Wu L, Feng J, Li J, Liu T, Zhang R, Xu S, Cheng K, Zhou Y, Zhou S, et al: In vitro and in vivo study of epigallocatechin-3-gallate-induced apoptosis in aerobic glycolytic hepatocellular carcinoma cells involving inhibition of phosphofructokinase activity. Sci Rep 6: 28479, 2016.

25. Livak KJ and Schmittgen TD: Analysis of relative gene expression data using real-time quantitative PCR and the 2(-Delta Delta C(T)) method. Methods 25: 402-408, 2001

26. Chen S, Morine Y, Tokuda K, Yamada S, Saito Y, Nishi M, Ikemoto T and Shimada M: Cancer-associated fibroblast-induced M2-polarized macrophages promote hepatocellular carcinoma progression via the plasminogen activator inhibitor-1 pathway. Int J Oncol 59: 59, 2021.

27. Fiori ME, Di Franco S, Villanova L, Bianca P, Stassi G and De Maria R: Cancer-associated fibroblasts as abettors of tumor progression at the crossroads of EMT and therapy resistance. Mol Cancer 18: 70, 2019.

28. Kim J and DeBerardinis RJ: Mechanisms and implications of metabolic heterogeneity in cancer. Cell Metab 30: 434-446, 2019.

29. Salem AF, Whitaker-Menezes D,LinZ, Martinez-Outschoorn UE, Tanowitz HB, Al-Zoubi MS, Howell A, Pestell RG, Sotgia F and Lisanti MP: Two-compartment tumor metabolism: Autophagy in the tumor microenvironment and oxidative mitochondrial metabolism (OXPHOS) in cancer cells. Cell Cycle 11: 2545-2556, 2012.

30. Witkiewicz AK, Whitaker-Menezes D, Dasgupta A, Philp NJ, Lin Z, Gandara R, Sneddon S, Martinez-Outschoorn UE, Sotgia F and Lisanti MP: Using the 'reverse Warburg effect' to identify high-risk breast cancer patients: Stromal MCT4 predicts poor clinical outcome in triple-negative breast cancers. Cell Cycle 11: 1108-1117, 2012. 
31. Pavlides S, Vera I, Gandara R, Sneddon S, Pestell RG, Mercier I, Martinez-Outschoorn UE, Whitaker-Menezes D, Howell A, Sotgia F and Lisanti MP: Warburg meets autophagy: Cancer-associated fibroblasts accelerate tumor growth and metastasis via oxidative stress, mitophagy, and aerobic glycolysis. Antioxid Redox Signal 16: 1264-1284, 2012.

32. Gonzalez CD, Alvarez S, Ropolo A, Rosenzvit C, Bagnes MF and Vaccaro MI: Autophagy, Warburg, and Warburg reverse effects in human cancer. BioMed Res Int 2014: 926729, 2014.

33. Wilde L, Roche M, Domingo-Vidal M, Tanson K, Philp N, Curry J and Martinez-Outschoorn U: Metabolic coupling and the Reverse Warburg Effect in cancer: Implications for novel biomarker and anticancer agent development. Semin Oncol 44: 198-203, 2017

34. Cruz-Bermúdez A, Laza-Briviesca R, Vicente-Blanco RJ, García-Grande A, Coronado MJ, Laine-Menéndez S, Alfaro C, Sanchez JC, Franco F, Calvo V, et al: Cancer-associated fibroblasts modify lung cancer metabolism involving ROS and TGF- $\beta$ signaling. Free Radic Biol Med 130: 163-173, 2019.

35. Shao S, Qin T, Qian W, Yue Y, Xiao Y, Li X, Zhang D, Wang Z, $\mathrm{Ma} \mathrm{Q}$ and Lei J: Positive feedback in Cav-1-ROS signaling in PSCs mediates metabolic coupling between PSCs and tumour cells. J Cell Mol Med 24: 9397-9408, 2020.

36. Zhang Z, Gao Z, Rajthala S, Sapkota D, Dongre H, Parajuli H, Suliman S, Das R, Li L, Bindoff LA, et al: Metabolic reprogramming of normal oral fibroblasts correlated with increased glycolytic metabolism of oral squamous cell carcinoma and precedes their activation into carcinoma associated fibroblasts. Cell Mol Life Sci 77: 1115-1133, 2020.

37. Zhang X, Dong Y, Zhao M, Ding L, Yang X, Jing Y, Song Y, Chen S, Hu Q and Ni Y: ITGB2-mediated metabolic switch in CAFs promotes OSCC proliferation by oxidation of $\mathrm{NADH}$ in mitochondrial oxidative phosphorylation system. Theranostics 10: 12044-12059, 2020.

38. Sung JS, Kang CW, Kang S, Jang Y, Chae YC, Kim BG and Cho NH: ITGB4-mediated metabolic reprogramming of cancer-associated fibroblasts. Oncogene 39: 664-676, 2020.

39. Ippolito L, Morandi A, Taddei ML, Parri M, Comito G, Iscaro A Raspollini MR, Magherini F, Rapizzi E, Masquelier J, et al: Cancer-associated fibroblasts promote prostate cancer malignancy via metabolic rewiring and mitochondrial transfer. Oncogene 38: 5339-5355, 2019.

40. Wu X, Zhou X, Xu S, Liao C, Chen X, Li B, Peng J, Li D and Yang L: Extracellular vesicle packaged LMP1-activated fibroblasts promote tumor progression via autophagy and stroma-tumor metabolism coupling. Cancer Lett 478: 93-106, 2020.

41. Deng L, Jiang N, Zeng J, Wang Y and Cui H: The versatile roles of cancer-associated fibroblasts in colorectal cancer and therapeutic implications. Front Cell Dev Biol 9: 733270, 2021.
42. Mao X, Xu J, Wang W, Liang C, Hua J, Liu J, Zhang B, Meng Q, Yu X and Shi S: Crosstalk between cancer-associated fibroblasts and immune cells in the tumor microenvironment: New findings and future perspectives. Mol Cancer 20: 131, 2021.

43. Kobayashi H, Enomoto A, Woods SL, Burt AD, Takahashi M and Worthley DL: Cancer-associated fibroblasts in gastrointestinal cancer. Nat Rev Gastroenterol Hepatol 16: 282-295, 2019.

44. Tan HX, Gong WZ, Zhou K, Xiao ZG, Hou FT, Huang T, Zhang L, Dong HY, Zhang WL, Liu Y, et al: CXCR4/TGF- $\beta 1$ mediated hepatic stellate cells differentiation into carcinoma-associated fibroblasts and promoted liver metastasis of colon cancer. Cancer Biol Ther 21: 258-268, 2020.

45. Wu YH, Huang YF, Chang TH, Chen CC, Wu PY, Huang SC and Chou CY: COL11A1 activates cancer-associated fibroblasts by modulating TGF- $\beta 3$ through the NF- $\mathrm{KB} / \mathrm{IGFBP} 2$ axis in ovarian cancer cells. Oncogene 40: 4503-4519, 2021.

46. Park CR, Lee JS, Son CG and Lee NH: A survey of herbal medicines as tumor microenvironment-modulating agents. Phytother Res 35: 78-94, 2021

47. Whitaker-Menezes D, Martinez-Outschoorn UE, Lin Z, Ertel A, Flomenberg N, Witkiewicz AK, Birbe RC, Howell A, Pavlides S, Gandara R, et al: Evidence for a stromal-epithelial 'lactate shuttle' in human tumors: MCT4 is a marker of oxidative stress in cancer-associated fibroblasts. Cell Cycle 10: 1772-1783, 2011.

48. Fiaschi T, Marini A, Giannoni E, Taddei ML, Gandellini P, De Donatis A, Lanciotti M, Serni S, Cirri P and Chiarugi P: Reciprocal metabolic reprogramming through lactate shuttle coordinately influences tumor-stroma interplay. Cancer Res 72: 5130-5140, 2012

49. Sun K, Tang S, Hou Y, Xi L, Chen Y, Yin J, Peng M, Zhao M, Cui X and Liu M: Oxidized ATM-mediated glycolysis enhancement in breast cancer-associated fibroblasts contributes to tumor invasion through lactate as metabolic coupling. EBioMedicine 41: 370-383, 2019.

50. Futagi Y, Kobayashi M, Narumi K, Furugen A and Iseki K Identification of a selective inhibitor of human monocarboxylate transporter 4. Biochem Biophys Res Commun 495: 427-432, 2018.

51. Hui S, Ghergurovich JM, Morscher RJ, Jang C, Teng X, Lu W, Esparza LA, Reya T, Le Zhan, Yanxiang Guo J, et al: Glucose feeds the TCA cycle via circulating lactate. Nature 551: 115-118, 2017.

52. Kim BG, Sung JS, Jang Y, Cha YJ, Kang S, Han HH, Lee JH and Cho NH: Compression-induced expression of glycolysis genes in CAFs correlates with EMT and angiogenesis gene expression in breast cancer. Commun Biol 2: 313, 2019.

This work is licensed under a Creative Commons Attribution-NonCommercial-NoDerivatives 4.0 International (CC BY-NC-ND 4.0) License. 